\title{
INFRARED PROPERTIES OF THE HALO, Bulge AND Disk OF THE EDGE-ON GALAXY NGC 7814
}

Ellen Simmat and Richard J. Tuffs

Max-Planck-Institut für Kernphysik, Heidelberg, Germany

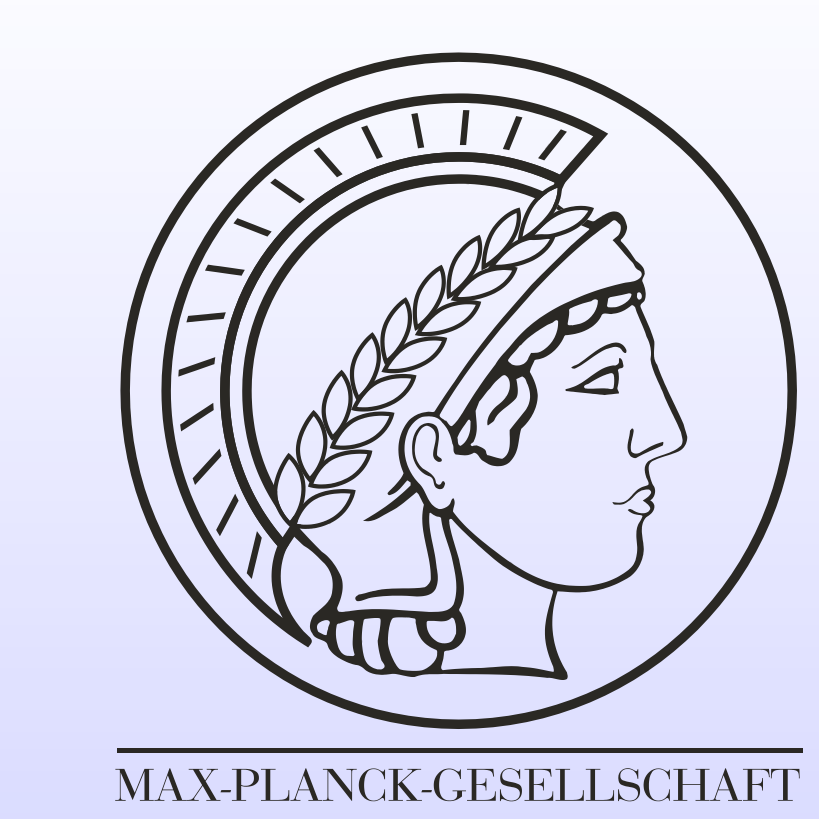

Highly resolved images of the edge-on galaxy NGC 7814 from 2MASS and Spitzer were used to extract the morphologies and spectral energy distributions (SEDs) of the disk, bulge and halo components of this galaxy over a wavelength range from 1.25 to $24 \mu \mathrm{m}$. This represents the first direct determination of the mid-infrared (MIR) emission of the bulge and halo components of a galaxy other than the Milky Way. Our analysis was performed in three steps. First, a structural decomposition was performed at each of the eight wavelengths (2MASS $1.25 \mu \mathrm{m}, 1.65 \mu \mathrm{m}, 2.17 \mu \mathrm{m}$; IRAC $3.6 \mu \mathrm{m}, 4.5 \mu \mathrm{m}, 5.8 \mu \mathrm{m}, 8.0 \mu \mathrm{m}$; MIPS $24 . \mu \mathrm{m}$; see box 1). Secondly, a spectral decomposition of the resulting halo, bulge and disk SEDs was performed (see box 2). The SEDs of all three structures imply the presence of emission components from direct stellar light and from at least one further component. For the disk, this further component is interpreted as dust emission having properties typical of star forming disks. The emission from the bulge and the halo is very significantly in excess of that from an extrapolation of the near infrared emission from stars. This excess has a flat spectrum between 8 and $24 \mu \mathrm{m}$ bands and its origin is unknown. Potential explanations are discussed in box 3.

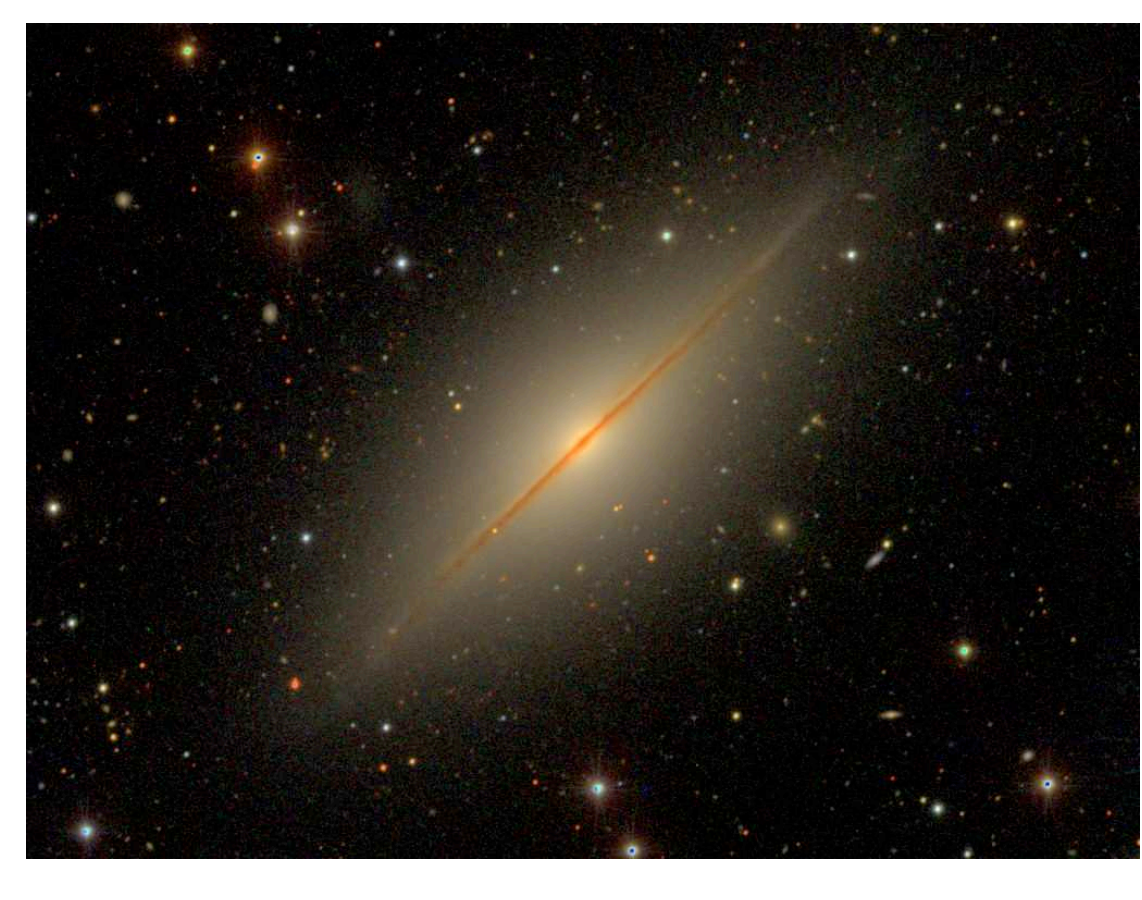

FIGURE 1: JHK false-colour imge of NGC 7814 from 2MASS The disk, the central bulge and the extended halo morphological components are clearly seen.

\section{Structural Decomposition of NGC 7814}

After subtracting the background, a structural decomposition was performed for each band in two steps: a 2D fit to the halo followed (after halo removal) by a $1 \mathrm{D}$ fit to the bulge and the disk

Halo fit in 2D

A 2D halo template was fitted to the images (Fig. 2 left for the example of the bands $3.6 \mu \mathrm{m}$ and $8 \mu \mathrm{m}$ ) in a region above and below the disk, where bulge and disk emission is negligible. We used a Sersic profile (Sérsic 1963) as radial emission profile with the fit parameters Sersic index, scale length, ellipticity and amplitude. The found halo model (middle) was removed from the data (right).
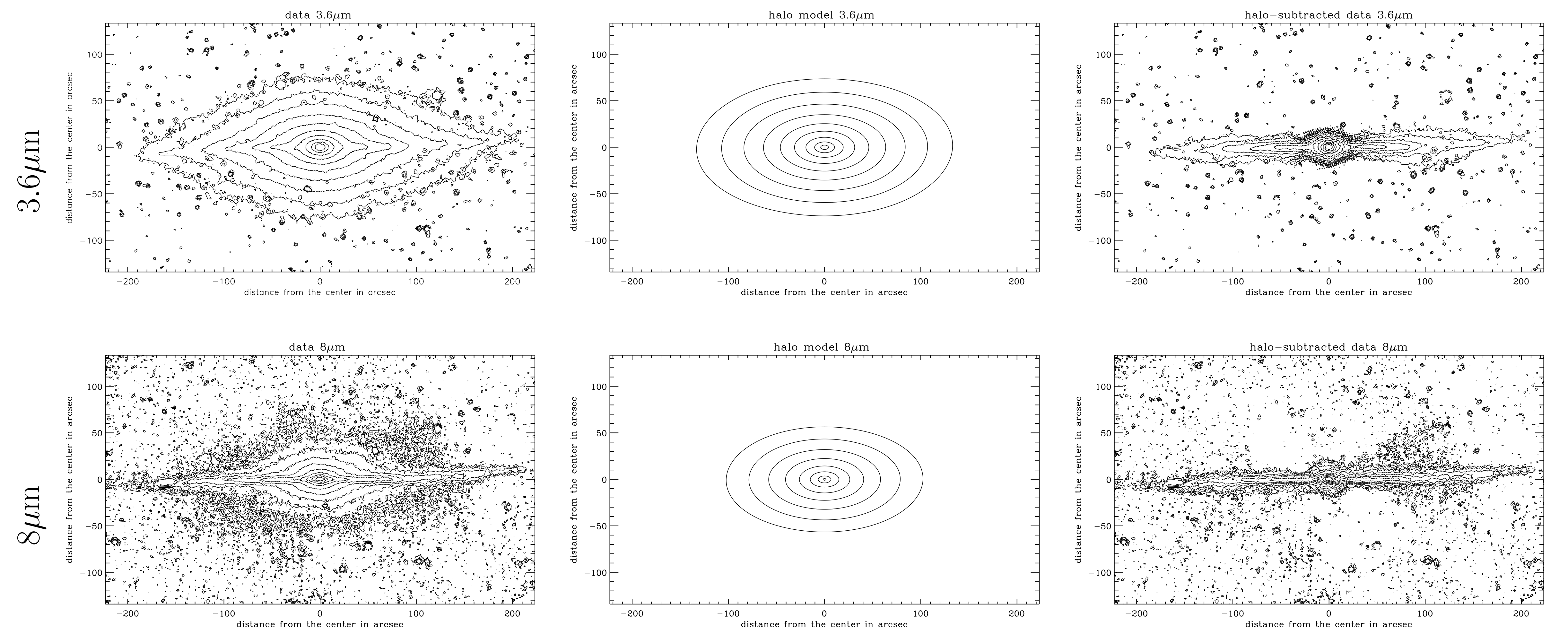

FiguRE 2: 2D fit to the halo for the example bands $3.6 \mu \mathrm{m}$ and $8 \mu \mathrm{m}$. Left: background-removed and rotated data, middle: fitted halo template, right: halo-removed

Bulge and disk fit in 1D
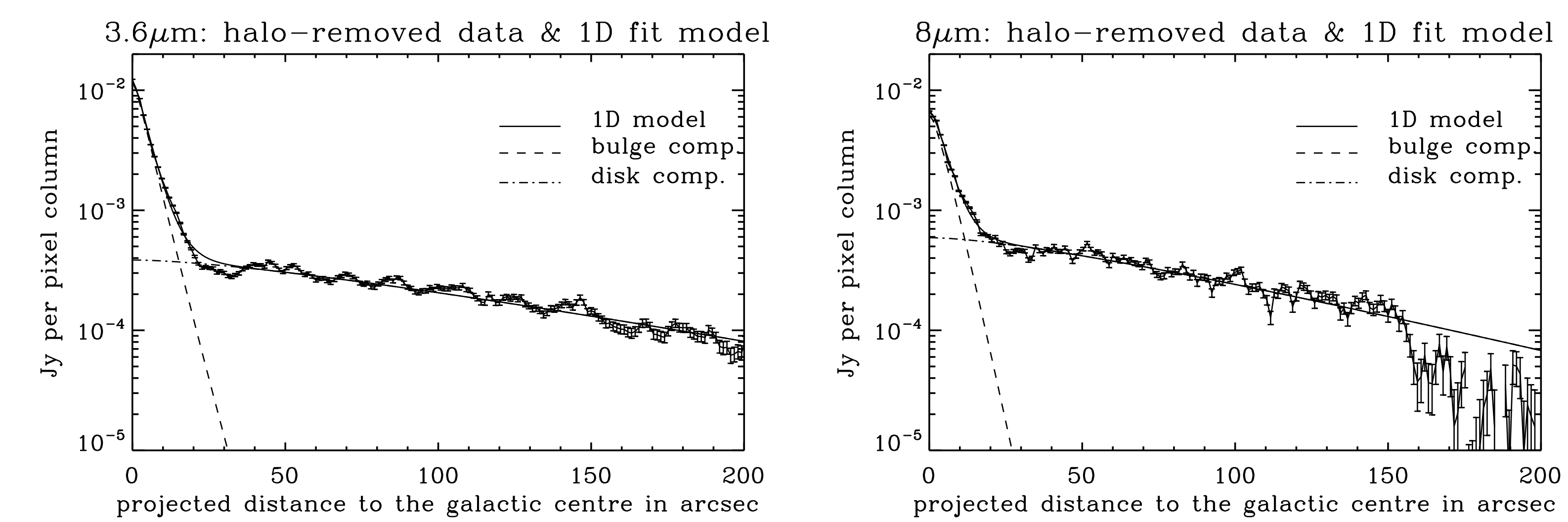

FIGURE 3: 1D profiles with the bulge and disk fit for the example bands $3.6 \mu \mathrm{m}$ and $8 \mu \mathrm{m}$
For the 1D fit to the bulge and the disk, the halo-removed data was integrated perpendicular to the disk and averaged on both sides of the galactic center, creating a projected radial profile of the edge-on galaxy-A superposition of an exponential (disk) and a Sersic profle (bulge), integrated along the line of sight in order to account for the inclination angle of the galaxy, was used to fit the morphological components. Fig. 3 illustrates these fits for the example bands $3.6 \mu \mathrm{m}$ and $8 \mu \mathrm{m}$. Note the fall off in the $8 \mu \mathrm{m}$ emission in the outer disk, a common feature of PAHemission from the diffuse ISM of spiral galaxies.
2. Spectral decomposition of the SEDs of halo, bulge and disk

SED of halo, bulge and disk

Integrating the fitted morphological components, we derived an SED or each of the halo, the bulge and the disk (Fig. 3).

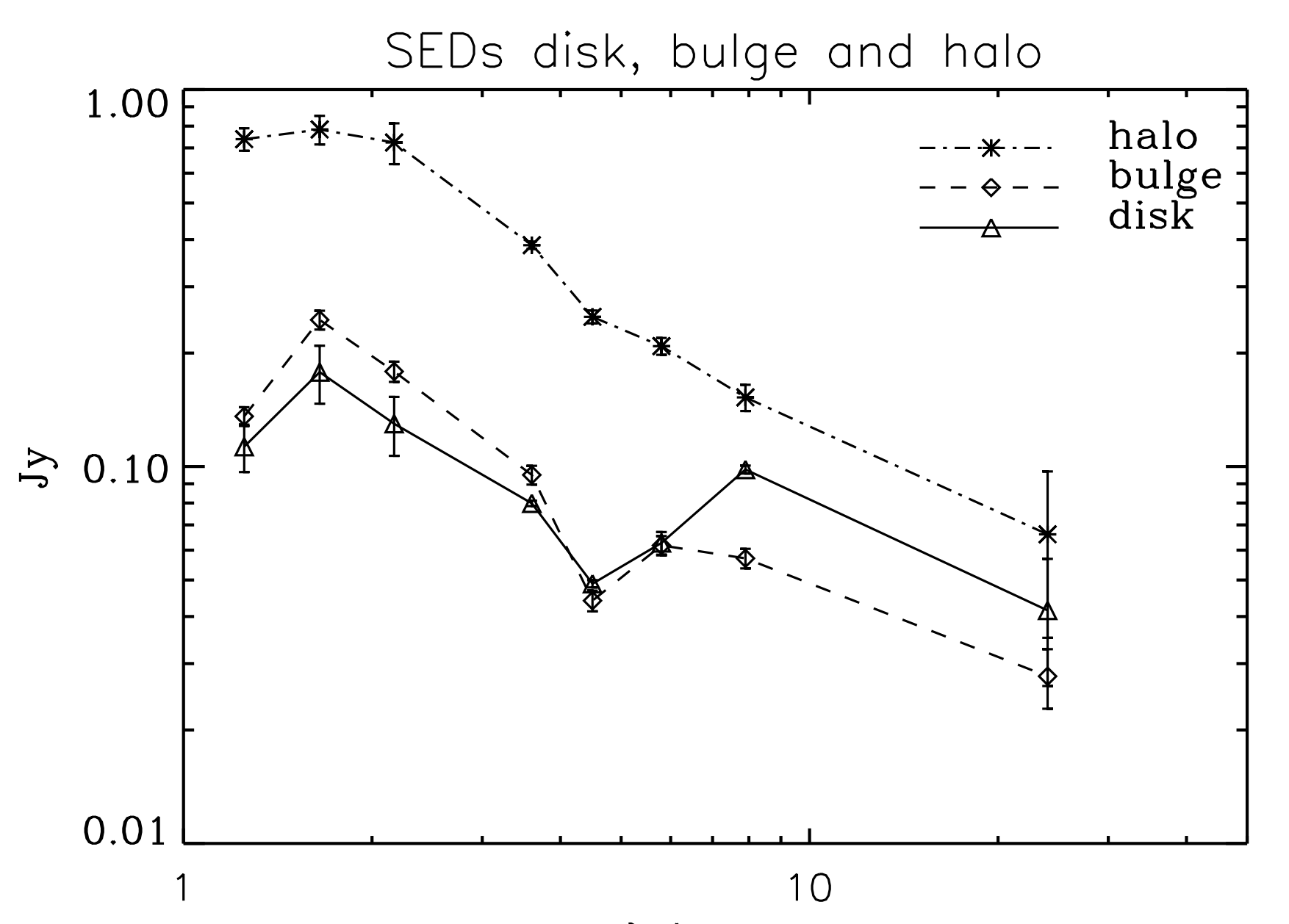

FIGURE 4: SED of halo, bulge and dist

Starlight removal of the halo

The SEDs in Fig. 4 are dominated by direct stellar light in the NIR. In order to remove this emission component, the bulge and disk SEDs were first corrected for attenuation due to dust in the disk following Tuffs et al. (2004), and then a population synthesis model constructed from Single age Stellar Populations (SSPs) by Cordier (2007) was fitted to all three SEDs in the NIR. Stellar age dors metallicity of the stellar population was assumed to be solar $(z=0.02)$ for the disk and lower $(\mathrm{z}=0.004)$ for the bulge and the halo. Fig. illustrates the fit of the halo and shows the resulting MIR residual.

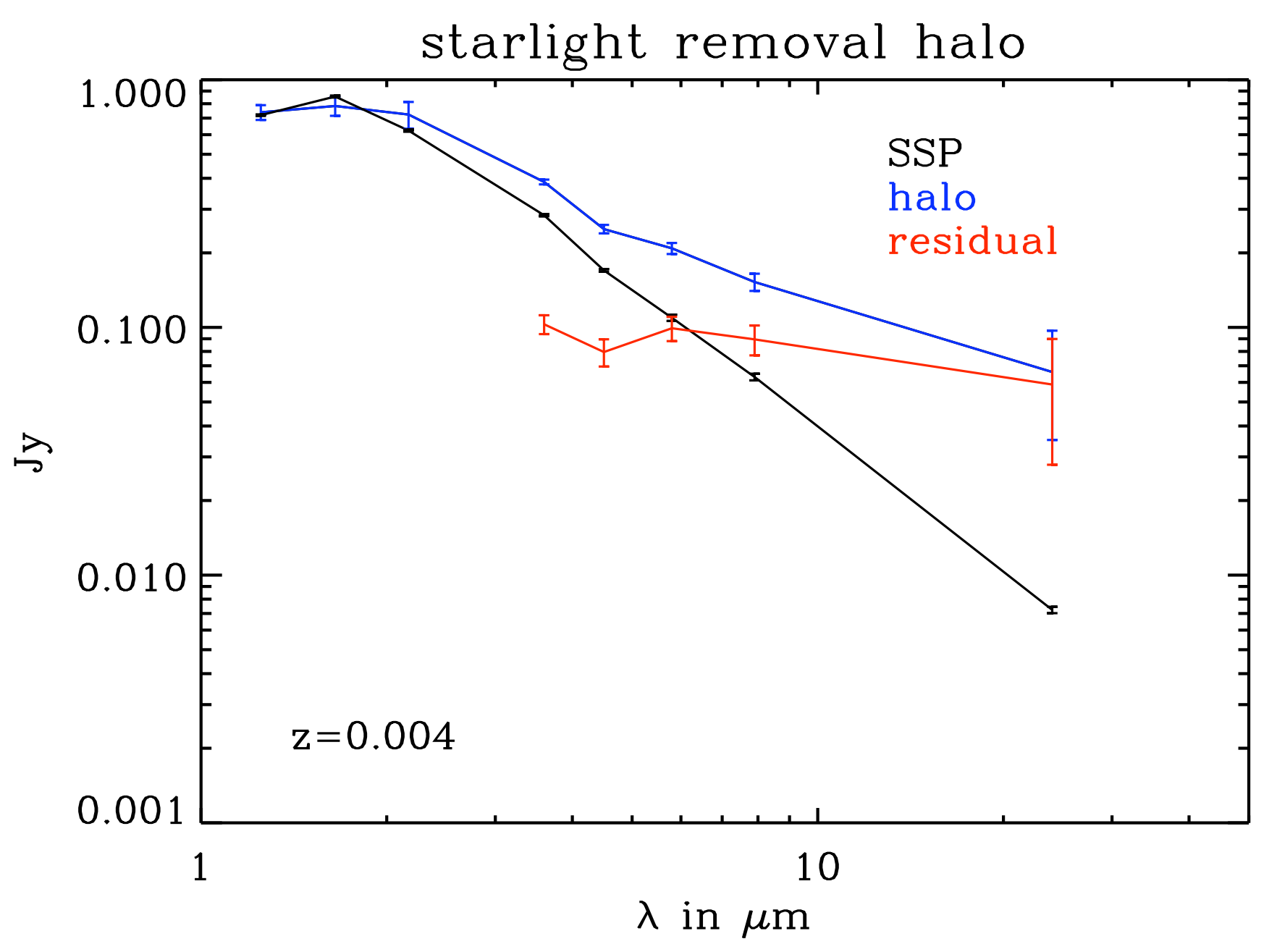

\section{Residual SED and possible sources}

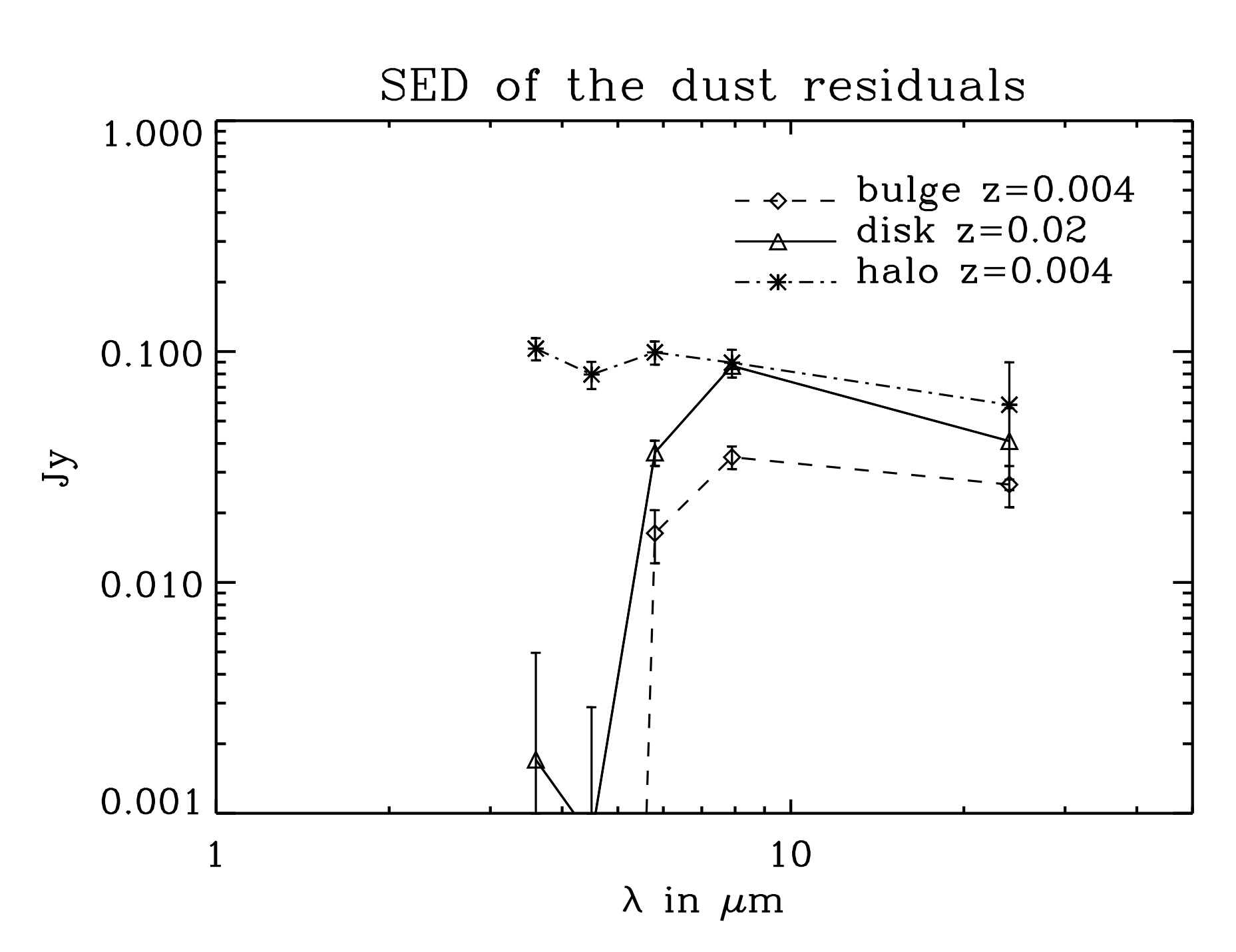

FIGURE 6: residual MIR SEDs
The residual SEDs after the starlight removal are shown in Fig. 6. All three morphological components show significant excess emission in the MIR. The halo has rather flat residual SED; the SEDs of disk and bulge show a very similar behavior with a peak at $8 \mu \mathrm{m}$. In case of the disk this excess emission is the thermal emission of the interstellar dust. We consider the following potential origins for the excess MIR emission of the halo and the bulge:

A) AGB Stars \& Circumstellar Dust

B) Interstellar Dust

C) Localized Star Formation in Galactic Fountains and Galactic Winds

D) Brown Dwarfs and Jupiters

Since the spatial distribution of the excess MIR emission follows that of the NIR stellar light, and since there is no counterpart in H $\alpha$ (Hameed \& Devereux 2005 ) or in X-ray (Halderson et al. 2001), possibility B and C are disfavored. However, in case of the bulge, an additional heating of the interstellar dust in the disk by the bulge stars could cause an excess of emission in the MIR. Substellar objects such as brown dwarfs or Jupiters are disfavored because a too high mass would be needed to account for the MIR excess. Therefore, we favour possibility A, thermal emission from circumstellar dust of AGB stars, with potentially minor contribution from the other possibilities.

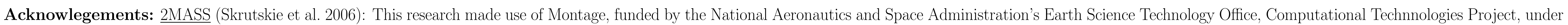
Cooperative Agreement Number NCC5-626 between NASA and the California Institute of Technology. The code is maintained by the NASA/IPAC Infrared Science Archive.

\section{References}

Cordier, D., Pietrinferni, A., Cassisi, S., \& Salaris, M. 2007, AJ, 133, 468

Halderson, E. L., Moran, E. C., Filippenko, A. V., \& Ho, L. C. 2001, AJ, 122, 637

\section{Hameed, S. \& Devereux, N. 2005, AJ, 129, 2597}

Sérsic, J. L. 1963, Boletin de la Asociacion Argentina de Astronomia La Plata Argentina, 6, 41

Skrutskie, M. F., Cutri, R. M., Stiening, R., et al. 2006, AJ, 131, 1163

Tuffs, R. J., Popescu, C. C., Völk, H. J., Kylafis, N. D., \& Dopita, M. A. 2004, A\&A, 419, 821 


\title{
Infrared Properties of the Halo, Bulge and Disk of the Edge-on Galaxy NGC 7814
}

\author{
Ellen Simmat and Richard J. Tuffs \\ Max-Planck-Institut für Kernphysik, Germany
}

\begin{abstract}
2MASS and Spitzer images of the edge-on galaxy NGC 7814 were used to extract the morphologies and spectral energy distributions (SEDs) of the disk, bulge and halo components of this galaxy from 1.25 to $24 \mu \mathrm{m}$. This represents the first direct determination of the mid-infrared emission of the bulge and halo components of a galaxy other than the Milky Way. The SEDs of all three structures imply the presence of emission components from direct stellar light and from at least one further component. For the disk, this is interpreted as dust emission having properties typical of star forming disks. The very significant excess emission from the bulge and the halo has an unknown origin. We discuss potential explanations such as dust emission in an interstellar medium, galactic winds, star forming regions and circumstellar dust, as well as emission from brown dwarfs.
\end{abstract}

\section{Deriving halo, bulge and disk SEDs}

\section{Morphological decomposition}

A 2D halo template was fitted to the disk emission-free areas of the 2MASS and Spitzer images of NGC 7814 (Fig. 1a) using a Sérsic profile with fit parameter Sérsic index, scale length, ellipticity and amplitude. The found halo model (Fig.1b) was then removed from the data to yield the image shown in Fig.1c. This image was reduced to a $1 \mathrm{D}$ radial profile (Fig.1d) which was fitted with a superposition of an exponential (disk) and a second Sérsic profile (bulge). This morphological decomposition procedure was applied at all 8 wavelengths, allowing SEDs from 1.2 to $24 \mu \mathrm{m}$ of the spatially integrated emission from the halo, bulge and disk components to be constructed. The SED of the halo is shown as the solid line in Fig.1e.

\section{Spectral decomposition of the halo, bulge and disk SEDs}

The SEDs of the halo, bulge and disk are dominated by direct stellar light in the NIR. In order to remove this emission component, the bulge and disk SEDs were first corrected for attenuation due to dust in the disk (using (Tuffs et al. 2004)). The halo template is not affected by attenuation caused by disk dust, since it was derived above and below the disk only. Then, the amplitude of a population synthesis model constructed from single age stellar population templates (Cordier et al. 2007) is fitted to the SEDs in the NIR. Stellar age does not change the model SED shape in the NIR and MIR regime. The metallicity of the stellar population is assumed to be solar for the disk and lower for the bulge and the halo. Fig.1e illustrates the fit of the halo and shows the resulting MIR residual and Fig.1f shows the residuals for halo, bulge and disk. 


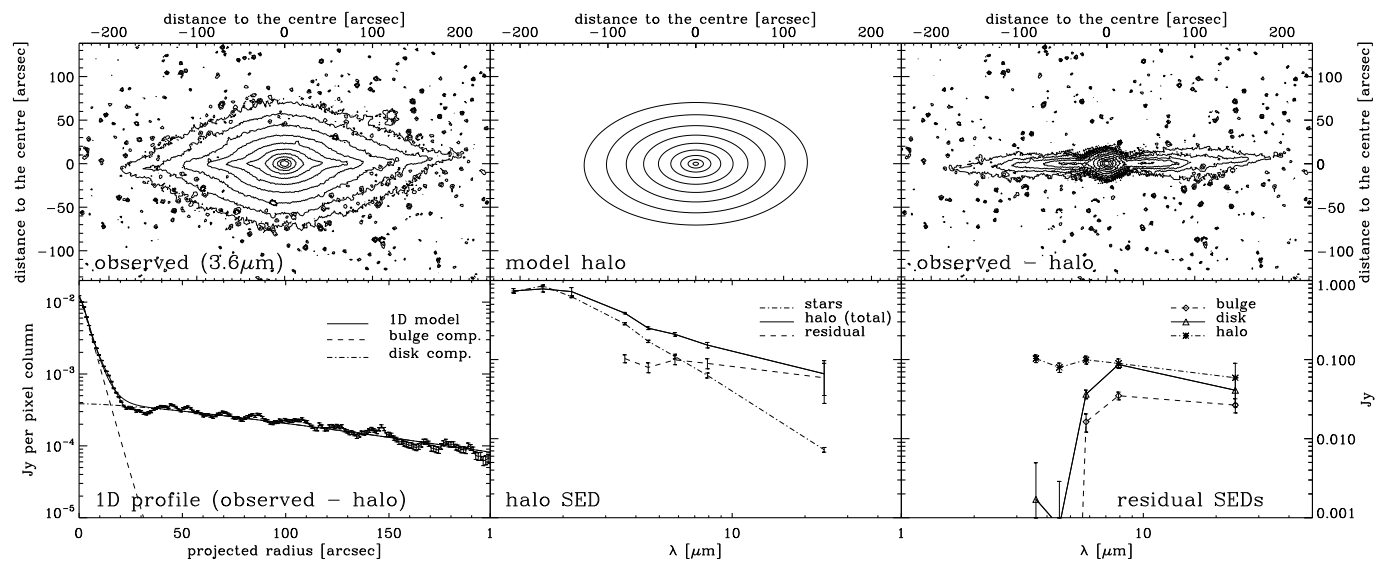

Figure 1: Top row, left to right: Morphological decomposition (example: $3.6 \mu \mathrm{m})$ : (a) data, (b) halo fit, (c) residual. Bottom row, left to right: (d) 1D reduction of the residuum with bulge and disk fit, (e) amplitude fit of the starlight SED template for the halo to the attenuation corrected NIR (JHK-band) SED, (f) residual SEDs after starlight removal for all three morphological components halo, bulge and disk.

\section{Residual SEDs: origin of the MIR excess from bulge and halo}

All three morphological components show significant excess emission in the MIR. The halo has a rather flat residual SED; the SEDs of disk and bulge show a very similar behaviour with a peak at $8 \mu \mathrm{m}$. The excess emission of the disk is interpreted as the emission of the interstellar dust. We consider the following potential origins for the excess MIR emission of the halo and the bulge: A) AGB stars \& circumstellar dust, B) interstellar dust, C) localized star formation in galactic fountains and galactic winds and D) substellar objects. Since the spatial distribution of the excess MIR emission follows that of the NIR stellar light, and since there is no counterpart in $\mathrm{H} \alpha$ (Hameed \& Devereux 2005) or in X-ray (Halderson et al. 2001), possibility B and C are disfavoured. However, part of the excess MIR emission of the bulge may be due to the enhanced heating of interstellar dust in the plane of the galaxy by bulge stars. Substellar objects such as brown dwarfs or Jupiters are disfavoured because a too high mass would be needed to account for the MIR excess. Therefore, we favour A, the thermal emission from circumstellar dust of AGB stars, as the main origin.

Acknowlegements 2MASS (Skrutskie et al. 2006): This research made use of Montage, funded by the NASA's Earth Science Technology Office, Computational Technnologies Project, under Cooperative Agreement Number NCC5-626 between NASA and the Caltech. The code is maintained by the NASA/IPAC Infrared Science Archive.

\section{References}

Cordier, D., Pietrinferni, A., Cassisi, S., \& Salaris, M. 2007, AJ, 133, 468

Halderson, E. L., Moran, E. C., Filippenko, A. V., \& Ho, L. C. 2001, AJ, 122, 637

Hameed, S. \& Devereux, N. 2005, AJ, 129, 2597

Skrutskie, M. F., Cutri, R. M., Stiening, R., et al. 2006, AJ, 131, 1163

Tuffs, R. J., Popescu, C. C., Völk, H. J., Kylafis, N. D., \& Dopita, M. A. 2004, A\&A, 419, 821 